\title{
ON TELESCOPING LINEAR EVALUATION FUNCTIONS
}

\author{
Ingo Althöfer \\ Fakultät für Mathematik \\ Universität Bielefeld \\ Germany
}

\section{THE RESULT}

Consider some finite set $X$, for instance the set of all legal chess positions. A real-valued function $f: X \rightarrow \mathbf{R}$ is called an evaluation function on $X$. In chess, one conventionally prefers evaluation functions that assume small values for positions unfavourable for White and large values for positions favourable for White.

Given $m$ arbitrary evaluation functions $f_{1}, \ldots, f_{m}$ on $X$, and real numbers $c_{1}, \ldots, c_{m}$, one can construct a new evaluation function $f$ on $X$ by taking

$f(x)=\sum_{i=1}^{m} c_{i} f_{i}(x) \quad$ for all positions $x$.

$f$ is called a linear combination of $f_{l}, \ldots, f_{m}$. Sometimes the $f_{i}$ are called elementary evaluation functions. A linear combination $f$ is called convex if the coefficients $c_{i}$ satisfy the following conditions:

$c_{i} \geq 0$ for all $i \quad$ and $\quad \sum_{i=1}^{m} c_{i}=1$.

In Althöfer (1993), I have proved the following theorem.

Theorem: $\quad$ Let $X$ be a finite set with $n$ elements. Let $f_{l}, \ldots, f_{m}$ be evaluation functions on $X$ with $0 \leq$ $f_{i}(x) \leq 1$ for $i=1, \ldots, m$ and all $x \in X$. Let $f=\sum_{i=1}^{m} c_{i} f_{i}$ be some convex combination of the $f_{i}$. Choose any constant $\varepsilon>0$ and let $k=\left\lceil\frac{\ln 2 n}{2 \varepsilon^{2}}\right\rceil$. Then there exists another convex combination $g=\sum_{i=1}^{m} d_{i} f_{i}$ of the same $f_{i}$ with the following properties:

(i) $|g(x)-f(x)| \leq \varepsilon$ for all $x \in X$

and

(ii) at most $k$ coefficients $d_{i}$ differ from zero. Moreover, the $d_{i}>0$ are of the form $d_{i}=k_{i} / k$ with natural numbers $k_{i}$.

$\lceil z\rceil$ denotes the ceiling, i.e., the smallest integer not smaller than the real number $z$.

Interpretation: (i) states that $g$ is an $\varepsilon$-approximation of $f$; (ii) states that the number of terms only grows logarithmically with the number of elements in the set $X$ and that the $d_{i}$ are rational numbers.

The operation of finding $k$ coefficients to represent a function which possibly required many more coefficients is known as telescoping. 
The theorem states the existence and does not describe a construction. However, a known result not proved here is that for every fixed $\varepsilon>0 \mathrm{~g}$ can be constructed by a probabilistic algorithm in expected time $O(n \mathrm{ln}$ $n$ ) (Althöfer, 1993). This means that the problem, being $O(n \ln n)$, is as hard as sorting which is also $O(n$ In $n$ ) (Knuth, 1973). Moreover, halving $\varepsilon$ increases computation time by a factor of 4 . Also note that the construction assumes that the simple $f_{i}(i=1, \ldots, m)$, the elementary evaluation functions, are given, which regrettably is not the case.

\section{APPLICATIONS TO CHESS AND OTHER GAMES}

It has been estimated that there are approximately $10^{53}$ many legal chess positions (Allis, Van den Herik and Herschberg, 1991). For convenience, in all the following examples the elementary evaluation functions $f_{i}$ are assumed to satisfy $0 \leq f_{i}(x) \leq 1$ for all positions $x \in X$.

\section{Example 1}

Consider the set $X$ of all legal chess positions and a convex combination $f=\sum_{i=1}^{m} c_{i} f_{i}$ of elementary evaluation functions $f_{i}$ which satisfies the following strong separation properties:

(i) $\quad 0 \leq f(x)<1 / 7 \quad$ if $x$ is a loss for White

(ii) $3 / 7<f(x)<4 / 7 \quad$ if $x$ is a drawn position

(iii) $6 / 7<f(x) \leq 1 \quad$ if $x$ is a win for White

Then there exists another (simple) convex combination $g=\sum_{i=1}^{m} d_{i} f_{i}$ of the same $f_{i}$ which satisfies the weaker separation properties

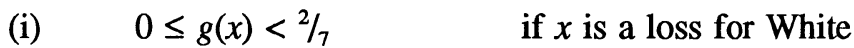

(ii) $2 / 7<g(x)<5 / 7 \quad$ if $x$ is a drawn position

(iii) $5 / 7<g(x) \leq 1 \quad$ if $x$ is a win for White

and

(iv) at most 3007 coefficients $d_{i}$ differ from zero.

Proof: Put cardinality $(X)=10^{53}, \varepsilon=1 / 7$, and apply the theorem.

\section{Example 2}

Consider some chess endgame (for instance with 5 pieces) with $10^{8}=100$ million legal positions $x$. Assume that there is some convex combination $f=\sum_{i=1}^{m} c_{i} f_{i}$ which satisfies the strong separation properties

(i) $\quad 0 \leq f(x)<1 / 4 \quad$ if $x$ is not a win for White

(ii) $3 / 4<f(x) \leq 1 \quad$ if $x$ is a win for White

Then there exists another (simple) convex combination $g=\sum_{i=1}^{m} d_{i} f_{i}$ of the same $f_{i}$ which satisfies the weaker separation properties

(i) $0 \leq g(x)<1 / 2 \quad$ if $x$ is not a win for White

(ii) $1 / 2<g(x) \leq 1 \quad$ if $x$ is a win for White

and

(iii) at most 153 coefficients $d_{i}$ differ from zero.

Proof: Put card $(X)=10^{8}, \varepsilon=1 / 4$, and apply the theorem. 


\section{Example 3}

Consider the chess endgame KBNNKR (Stiller and the Editors, 1991). There are fewer than $n=32 \times 63 \times$ $62 \times 61 \times 60 \times 59 \times 2$ legal positions in this endgame. Assume than there is some convex combination $f=$ $\sum_{i=1}^{m} c_{i} f_{i}$ which satisfies the strong separation properties

(i) $\quad 0 \leq f(x)<1 / 4 \quad$ if $x$ is not a win for White

(ii) $3 / 4<f(x) \leq 1 \quad$ if $x$ is a win for White.

Then there exists another (simple) convex combination $g=\sum_{i=1}^{m} d_{i} f_{i}$ of the same $f_{i}$ which satisfies the weaker separation properties

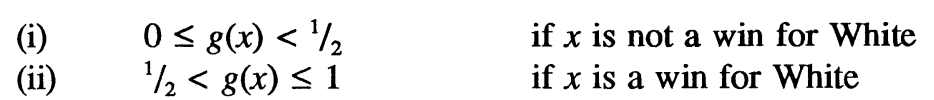

and

(iii) at most 204 coefficients $d_{i}$ differ from zero.

Proof: Put card $(X)=32 \times 63 \times 62 \times 61 \times 60 \times 59 \times 2 \approx 5.410^{10}, \varepsilon=1 / 4$ and apply the theorem.

\section{Example 4}

Consider the game of Go on a $19 \times 19$ board. Exploiting symmetries, there are fewer than $3^{361}$ legal positions. Assume that there is some convex combination $f=\sum_{i=1}^{m} c_{i} f_{i}$ of elementary evaluation functions $f_{i}$ which satisfies the strong separation properties

(i) $\quad 0 \leq f(x)<1 / 4 \quad$ if $x$ is not a win for Black

(ii) $3 / 4<f(x) \leq 1 \quad$ if $x$ is a win for Black

Then there exists another (simple) convex combination $g=\sum_{i=1}^{m} d_{i} f_{i}$ of the same $f_{i}$ which satisfies the weaker separation properties

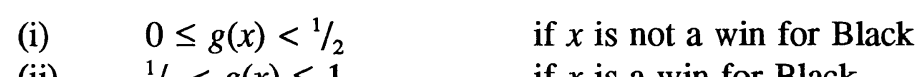

(ii) $1 / 2<g(x) \leq 1 \quad$ if $x$ is a win for Black

and

(iii) at most 3179 coefficients $d_{i}$ differ from zero.

Proof: Put card $(X)=3^{361}, \varepsilon=1 / 4$, and apply the theorem.

Of course the theorem can be applied also to all other finite games, for instance those investigated in Allis et al. (1991).

\section{CONCLUSIONS}

(i) Theorem 1 states: "if there is a convex combination $f=\sum c_{i} f_{i} \ldots$ then ...". It does not answer the question whether such an $f$ is so expressible; only if it is, the construction of $g=\sum d_{i} f_{i}$ from $f=\sum$ $c_{i} f_{i}$ becomes possible. In other words, without knowing the $c_{i}$ we may not construct the $d_{i}$.

(ii) Theorem 1 may be interpreted in the context of the search-versus-knowledge discussion: convex combinations of elementary evaluation functions with very many terms may often have shorter counterparts in which the deterioration of quality may be controlled. 
(iii) Of course one may argue that convex combinations are often not the best way to use (large) sets of elementary evaluation functions.

\title{
4. REFERENCES
}

Allis, L.V., Herik, H.J. van den and Herschberg, I.S. (1991) Which Games Will Survive? In: Heuristic Programming in Artificial Intelligence 2: The Second Computer Olympiad (eds. D.N.L. Levy and D.F. Beal), pp. 232-243. Ellis Horwood, Chichester.

Althöfer, I. (1993). On Sparse Approximations of Randomized Strategies and Convex Combinations. To appear in Linear Algebra and Its Applications.

Knuth, D.E. (1973). The Art of Computer Programming. Volume 3: Sorting and Searching. Addison-Wesley Publishing Company, Reading, MA.

Stiller, L. and the Editors (1991). Karpov and Kasparov: the End is Perfection. ICCA Journal, Vol. 14, No. 4, pp. 198-200.

\section{REVIEW}

\section{SECRETS OF ROOK ENDINGS}

\author{
by John Nunn \\ London, UK \\ B.T. Batsford Ltd., 1992 \\ London, UK \\ 320 pages \\ ISBN 0-7134-7164-6
}

Reviewed by H.J. van den Herik and I.S. Herschberg

The very title of John Nunn's most recent book reads more like a novel's and indeed it is a novel kind of book. The novelty is in its utter perfection and unassailable solidity. It is deeply satisfying to find the exclamation mark used with a precise, stated definition, instead of depending on a commentator's passing fancy. The query, too, now is perfectly objective. These are just two minor delights in a delightful major work showing "the almost inexhaustible nature of chess".

It must not be thought that this book is merely a digest of Ken Thompson's KRPKR database (Thompson, 1990), which later was made public on a first volume of a series of CD-ROMs (see Thompson, 1991). Lars Rasmussen was an important intermediary, providing a user-friendly interface which rendered the ultracompact database grandmaster-friendly. Needless to say, that the systematics of this book leaves nothing to be desired: its main subdivision is into Chapters, one each for the pawn files $a, b, c$, and d. Within each chapter, the subdivision is by pawn rank. Within that ranking, human terminology induces a further grouping according to whether the King or Rook is in front of or behind the Pawn, defends it from the side, and a miscellaneous section.

The book is enlivened by no fewer than 534 diagrams on 320 pages. Rolf Schlösser's Postscript chess fonts are uniform in text and diagrams, a clear case where computer typesetting contributes to the aesthetics of the printed page. 